\title{
ARCO AÓRTICO DIREITO COM ARTÉRIA SUBCLÁVIA ESOUERDA ABERRANTE E DIVERTÍCULO DE KOMMERELL*
}

\author{
Rafael Artigas Faucz ${ }^{1}$, Sérgio Furlan ${ }^{1}$, André Santos Barros ${ }^{1}$, Antônio Mauro Bof ${ }^{2}$, \\ Emílio Scafura Lomonte ${ }^{2}$, Luis Carlos Leiro ${ }^{3}$, Ricardo Pires de Souza ${ }^{4}$
}

\begin{abstract}
Resumo É relatado um caso de uma paciente do sexo feminino, 40 anos de idade, com queixas de disfagia e dor torácica retroesternal há três anos causadas pela presença de artéria subclávia esquerda aberrante retroesofágica com origem em uma dilatação aneurismática (divertículo de Kommerell). $\mathbf{O}$ arco aórtico e a aorta torácica descendente estão localizados à direita. 0 diagnóstico foi estabelecido por meio dos exames de esofagograma, tomografia computadorizada e angiorressonância magnética. A paciente apresentou melhora importante com o tratamento clínico e, atualmente, segue em acompanhamento ambulatorial há um ano.

Unitermos: Arco aórtico à direita; Artéria subclávia esquerda aberrante; Divertículo de Kommerell; Radiologia.
\end{abstract}

Abstract Right-sided aortic arch with aberrant left subclavian artery and Kommerell's diverticulum.

We report a case of a 40-year-old female patient presenting with dysphagia and retrosternal chest pain for three years caused by a retroesophageal aberrant left subclavian artery originating from an aneurysmatic dilatation (Kommerell's diverticulum). The aortic arch and descending thoracic aorta were right-sided. Diagnosis was established by esophagogram, computed tomography and magnetic resonance angiography. The patient was very responsive to clinical treatment and she is presently being followed-up in the outpatient clinic for over one year.

Key words: Right aortic arch; Aberrant left subclavian artery; Kommerell's diverticulum; Radiology.

\section{INTRODUÇÃO}

$\mathrm{O}$ arco aórtico à direita é um defeito congênito incomum da aorta. Sua primeira descrição foi feita há mais de dois séculos por Fioratti e Aglietti ${ }^{(\mathbf{1})}$. Diversas classificações foram propostas com base na distribuição anatômica das estruturas envolvidas, sendo a mais usada a classificação de Edwards ${ }^{(2)}$.

$\mathrm{O}$ arco aórtico à direita freqüentemente é assintomático, a menos que ocorra o desenvolvimento de um aneurisma. Isto, em geral, ocorre ao nível da origem da artéria subclávia esquerda e é conhecido como aneurisma de Kommerell ou divertículo de Kommerell, o qual pode ocasionar com-

* Trabalho realizado nos Departamentos de Radiologia e Diagnóstico por Imagem e de Cirurgia Torácica do Hospital Heliópolis, São Paulo, SP.

1. Médicos Residentes do Departamento de Radiologia e Diagnóstico por Imagem do Hospital Heliópolis.

2. Médicos Residentes do Departamento de Cirurgia Torácica do Hospital Heliópolis.

3. Médico Chefe do Departamento de Cirurgia Torácica do Hospital Heliópolis.

4. Médico Radiologista, Doutor em Medicina, Coordenador da Residência de Radiologia e Diagnóstico por Imagem do Hospital Heliópolis.

Endereço para correspondência: Dr. Rafael Artigas Faucz. Rua Doutor Diogo de Faria, 1201, ap. 87, Vila Mariana. São Paulo, SP, 04037-004. E-mail: rafaucz@terra.com.br

Recebido para publicação em 20/9/2004. Aceito, após revisão, em 9/12/2004. pressão de estruturas mediastinais e/ou romper espontaneamente.

O objetivo deste artigo é relatar um caso de arco aórtico à direita com artéria subclávia esquerda aberrante e divertículo de Kommerell, mostrando os aspectos de imagem no esofagograma, tomografia computadorizada e angiorressonância magnética.

\section{RELATO DO CASO}

Paciente do sexo feminino, 40 anos de idade, referia que há cerca de três anos iniciou com história de disfagia e dor torácica retroesternal. Negava hipertensão arterial e cardiopatia. Exame físico: $\mathrm{PA}=150 / 90$ $\mathrm{mmHg}, \mathrm{FC}=72 \mathrm{btm}$, bulhas cardíacas rítmicas, normofonéticas e sem sopros.

Foi realizada radiografia simples de tórax da paciente, que não evidenciou alterações. Endoscopia digestiva alta mostrou abaulamento póstero-anterior esofágico por compressão extrínseca. Esofagograma mostrou falha de enchimento em esôfago proximal (Figura 1). Tomografia computadorizada de tórax com janela de partes moles evidenciou aorta, incluindo o arco, localizada à direita da linha média e promovendo impressão no esôfago proximal e traqueobrônquico (Figura 2). Angiorres- sonância magnética 3D com gadolínio da aorta torácica demonstrou arco aórtico à direita com artéria subclávia esquerda aberrante que se originava de dilatação denominada de divertículo de Kommerell, com fluxo livre por toda a extensão estudada (Figura 3).

Iniciou-se tratamento clínico com o uso de medicação procinética e a paciente teve importante melhora clínica. Atualmente, ela segue assintomática em acompanhamento ambulatorial há um ano.

\section{DISCUSSÃO}

O arco aórtico direito é um defeito congênito raro da aorta e está presente em cerca de $0,05 \%$ da população ${ }^{(3)}$. Em uma série de autópsias, $50 \%$ dos casos foram associados com artéria subclávia esquerda aberrante $^{(4)}$, a qual pode estar localizada atrás do esôfago (80\%), entre a traquéia e o esôfago $(15 \%)$ ou anteriormente à traquéia $(5 \%)^{(5)}$.

Edwards $^{(2)}$ descreveu três tipos principais de arco aórtico à direita: tipo I, com os ramos das grandes artérias formando imagem em espelho (Figura 4A); tipo II, com uma artéria subclávia aberrante (Figura 4B); e tipo III, com artéria subclávia 


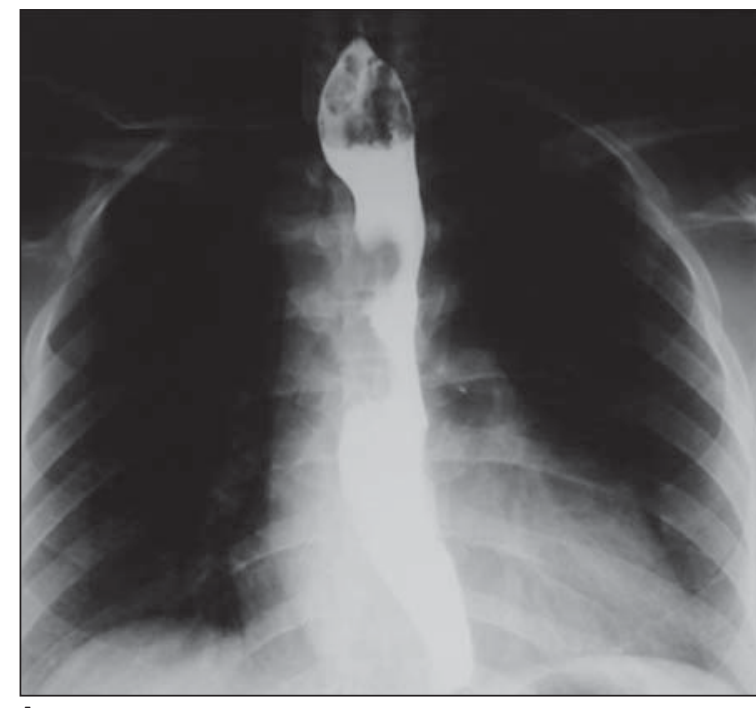

A

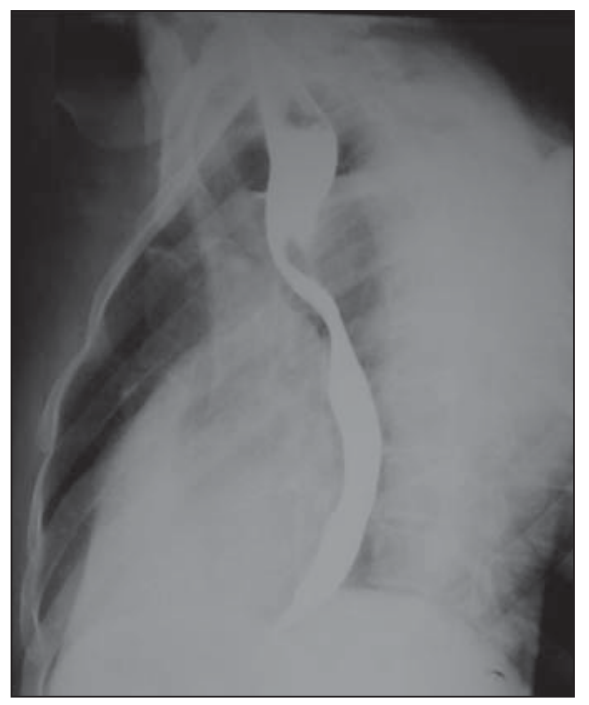

B
Figura 1. Esofagograma. Incidências ântero-posterior (A) e oblíqua (B) mostram falha de enchimento no esôfago proximal.

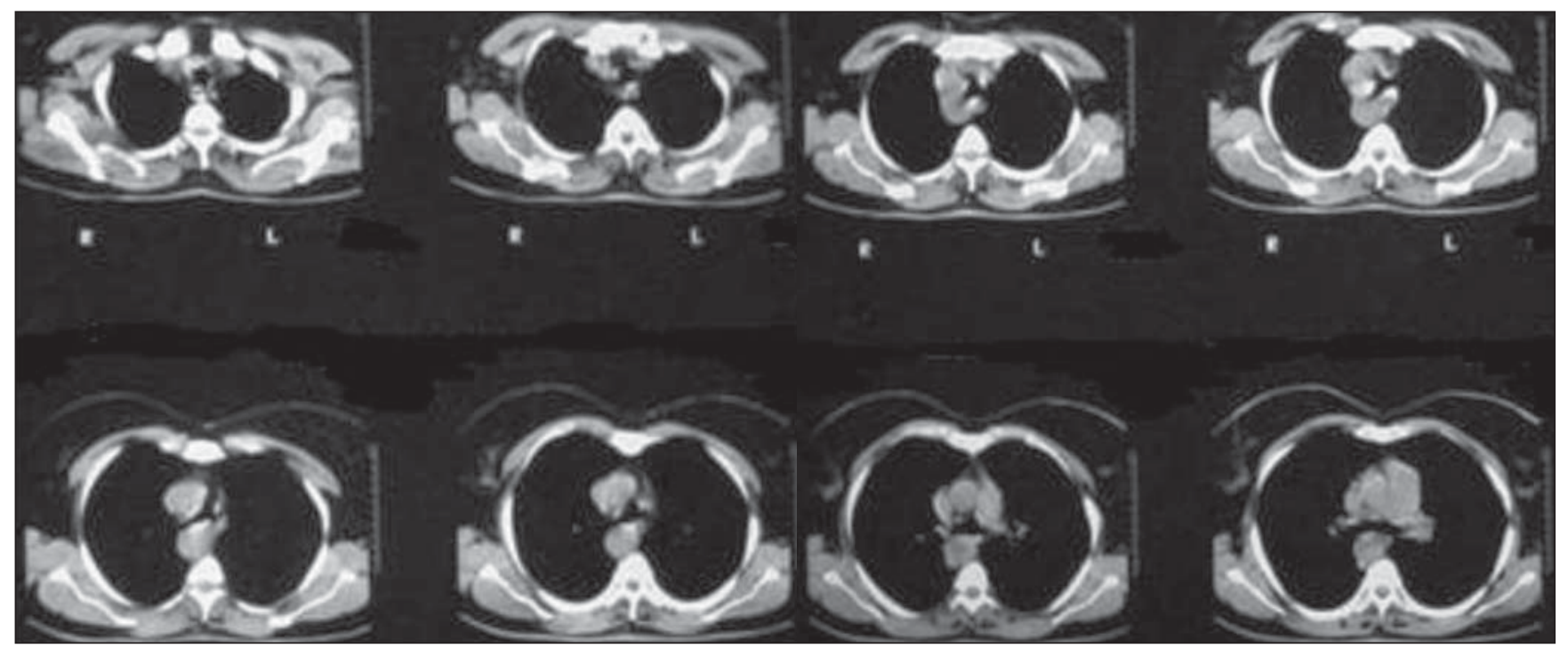

Figura 2. TC de tórax com janela de partes moles mostra arco aórtico e aorta torácica situados à direita da linha média promovendo impressão no esôfago proximal e traqueobrônquico.

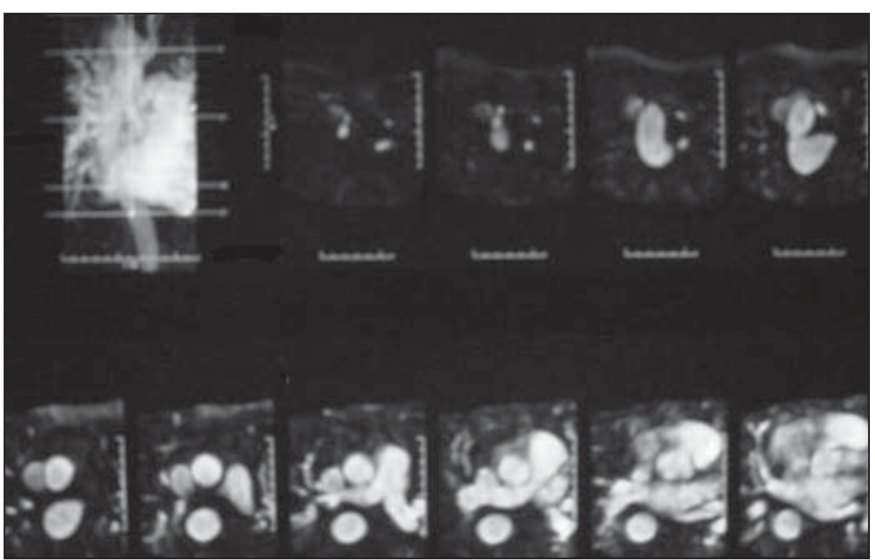

A (A,B,C). Ang oressonancia magnética 3D com gadolio mosta arco aortico a

A Figura 3 (A,B,C). Angioressonância magnética 3D com gadolnio mostra arco ao tículo de Kommerell. ASD, artéria subclávia direita; ASE, artéria subclávia esquerda.

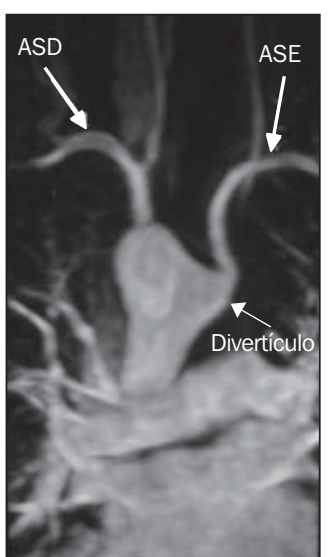

B

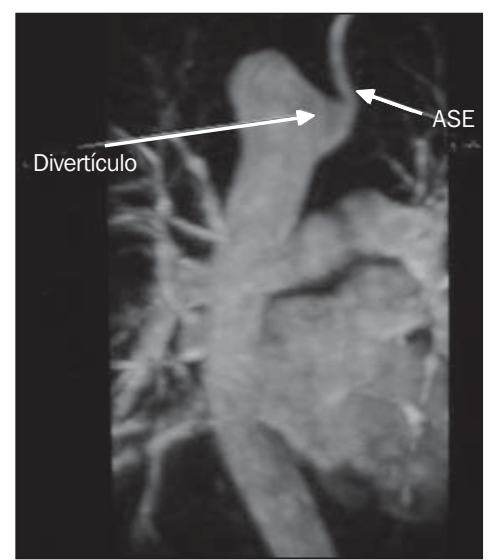

C 


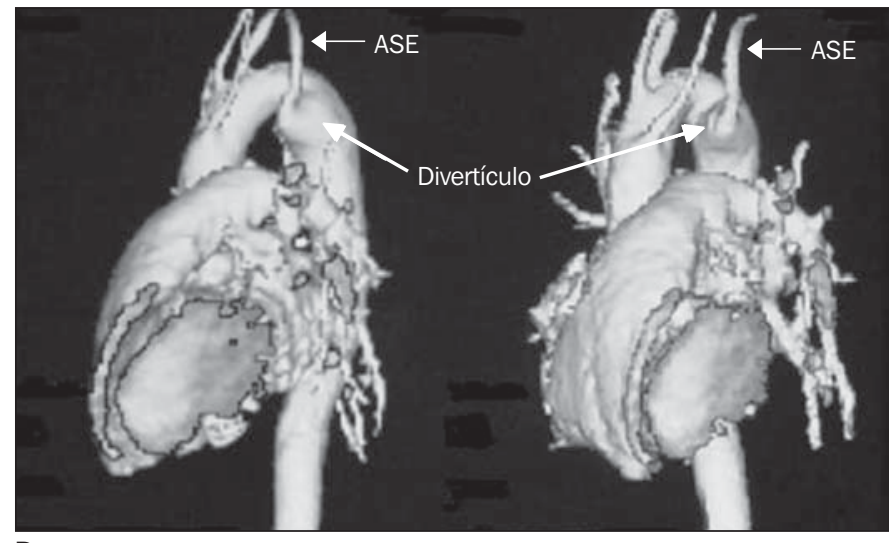

D

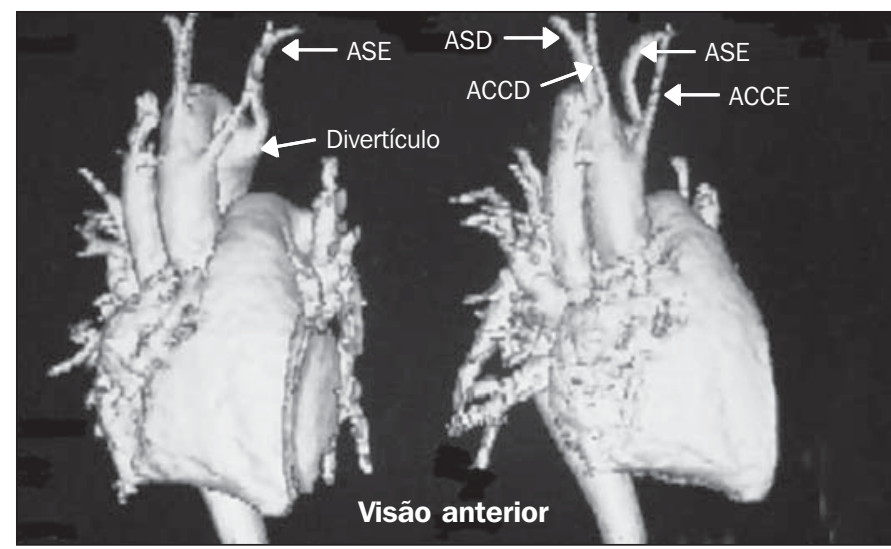

E

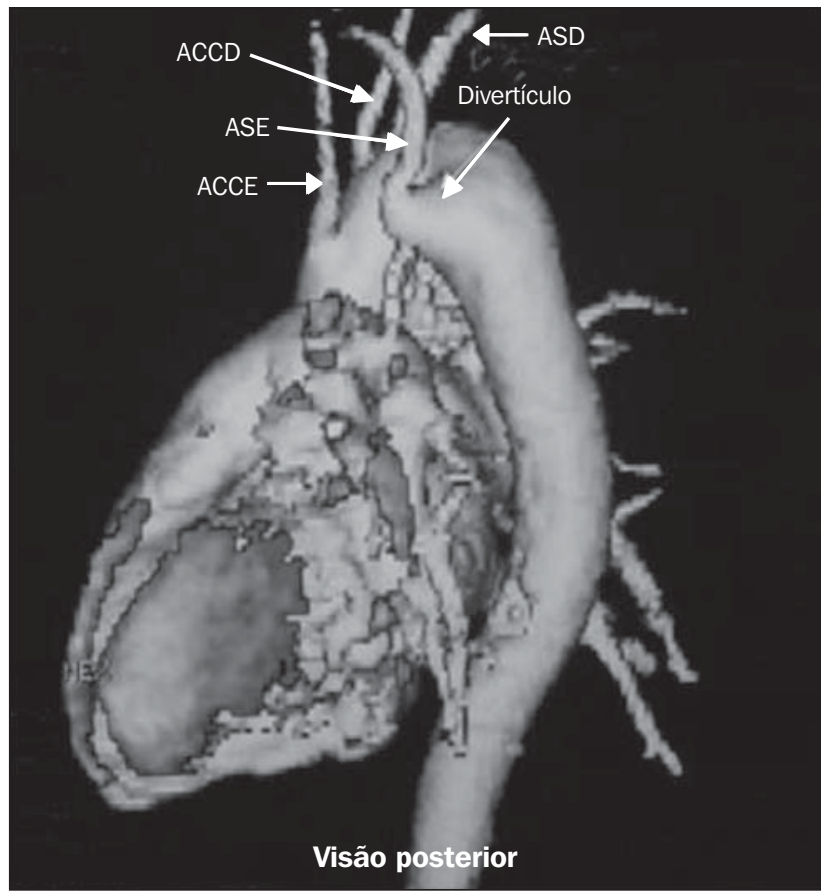

$\mathbf{F}$

Figura 3 (D,E,F). Angiorressonância magnética 3D com gadolínio mostra arco aórtico à direita com artéria subclávia esquerda aberrante que se origina no divertículo de Kommerell. ASD, artéria subclávia direita; ASE, artéria subclávia esquerda; ACCD, artéria carótida comum direita; ACCE, artéria carótida comum esquerda.

Figura 4. Classificação das anomalias do arco aórtico à direita. A: Tipo I, os ramos do arco com imagem em espelho. B: Tipo II, com artéria subclávia esquerda aberrante. C: Tipo III, com artéria subclávia esquerda isolada. (Reproduzido de Cinà et al. $\left.{ }^{(6)}\right)$.

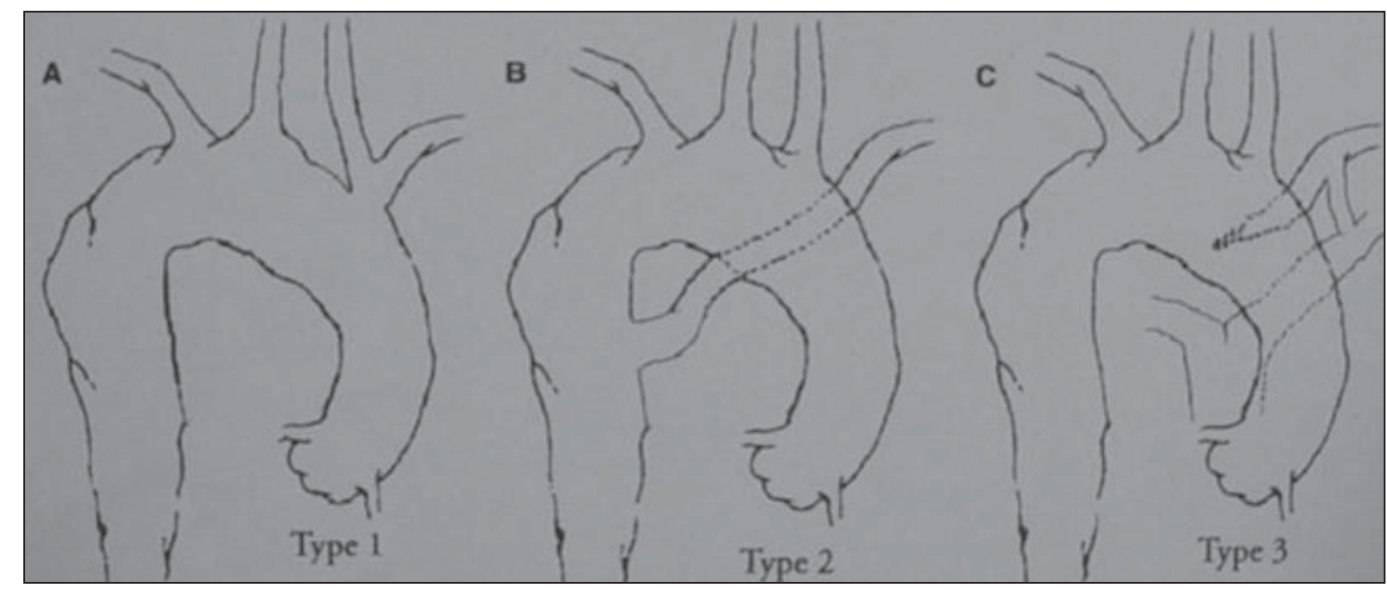

isolada (quando a artéria subclávia está conectada com a artéria pulmonar através do ductus arteriosus) (Figura 4C). Em nosso caso, a variante é classificada como do tipo II de Edwards, a qual representa 39,5\% de todos os casos de arco aórtico à direita ${ }^{(6)} \mathrm{e}$ em $5 \%$ a $10 \%$ dos casos está associada a cardiopatias congênitas (incluindo tetralo- gia de Fallot, estenose pulmonar com defeito septal ventricular, atresia tricúspide e ductus arteriosus) ${ }^{(\mathbf{6})}$.

A etiologia das anomalias do arco aórtico à direita é desconhecida. Uma deleção no cromossomo 22q11 está associada em $24 \%$ das anomalias isoladas de lateralidade dos ramos do arco aórtico ${ }^{(7)}$.
O arco aórtico direito é, freqüentemente, assintomático. Em crianças, os sintomas estão relacionados às anomalias cardíacas congênitas ou à compressão de estruturas mediastinais (traquéia e esôfago). Em adultos, sintomas resultam de alterações ateroscleróticas dos vasos anômalos, dissecção ou dilatação aneurismática com compressão 
das estruturas adjacentes causando disfagia (disfagia lusoria), dispnéia, estridor, respiração ruidosa, tosse, pneumonia de repetição, enfisema obstrutivo ou dor torácica ${ }^{(\mathbf{6})}$.

Ao nível da origem da artéria subclávia esquerda aberrante, raramente pode ocorrer o desenvolvimento de um aneurisma (aneurisma de Kommerell) $^{(\mathbf{8})}$. Um estudo de revisão da literatura relatou somente 32 casos descritos ${ }^{(\mathbf{9})}$. Desde então, poucos casos adicionais foram relatados ${ }^{(6,10-14)}$. Essa condição, também conhecida como divertículo de Kommerell, é clinicamente relevante devido ao aumento da mortalidade associada à sua ruptura, à morbidade causada pela compressão das estruturas mediastinais e à complexidade da cirurgia.

O tratamento, em geral, é cirúrgico e bastante complexo ${ }^{(\mathbf{1 0 , 1 2})}$. Os exames de imagem pré-operatórios são extremamente importantes para o planejamento da cirurgia, que depende da distribuição anatômica das estruturas locais, do tamanho e da extensão do aneurisma. Em nosso caso, porém, devido ao fato de a paciente ter apresentado excelente resposta ao uso da medicação procinética, optou-se pelo tratamento clínico e acompanhamento ambulatorial.

\section{REFERÊNCIAS}

1. Fioratti F, Aglietti F. A case of humam right aorta. Anatom Rec 1763;45:365.

2. Edwards JE. Anomalies of the derivatives of the aortic arch system. Med Clin N Am 1948;32:925948.

3. Svensson LG, Crawford ES. Congenital abnormalities of the aorta in adults. In: Svensson LG, Crawford ES, editors. Cardiovascular and vascular disease of the aorta. Philadelphia, Pa: WB Saunders, 1997;153-174.

4. Hastreiter AR, D'Cruz IA, Cantez T, Namin EP, Licata R. Right-sided aorta. Occurrence of right aortic arch in various types of congenital heart disease. Br Heart J 1966;28:722-725.

5. Gomes MM, Bernatz PE, Forth RJ. Arteriosclerotic aneurysm of an aberrant right subclavian artery. Dis Chest 1968;54:549-552.

6. Cinà CS, Althani H, Pasenau J, Abouzahr L. Kommerell's diverticulum and right-sided aortic arch: a cohort study and review of the literature. J Vasc Surg 2004;39:131-139.

7. McElhinney DB, Clark BJ III, Weinberg PM, et al.
Association of chromosome 22q11 deletion with isolated anomalies of aortic arch laterality and branching. J Am Coll Cardiol 2001;37:2114-2119.

8. Clagett JP. Upper extremity aneurysm. In: Rutherford RB, editor. Vascular surgery. Philadelphia, $\mathrm{Pa}$ WB Saunders, 1989;962.

9. Austin EH, Wolfe WG. Aneurysm of aberrant subclavian artery with a review of the literature. J Vasc Surg 1985;2:571-577.

10. Caus T, Gaubert JY, Monties JR, et al. Right-sided aortic arch: surgical treatment of an aneurysm arising from a Kommerell's diverticulum and extending to the descending thoracic aorta with an aberrant left subclavian artery. Cardiovasc Surg 1994;2: 110-113.

11. Cinà CS, Arena GO, Bruin G, Clase CM. Kommerell's diverticulum and aneurysmal right-sided aortic arch: a case report and review of the literature. J Vasc Surg 2000;32:1208-1214.

12. Tsukube T, Ataka K, Sakata M, Wakita N, Okita Y Surgical treatment of an aneurysm in the right aortic arch with aberrant left subclavian artery. Ann Thorac Surg 2001;71:1710-1711.

13. Esposito RA, Khalil I, Galloway AC, Spencer FC Surgical treatment for aneurysm of aberrant subclavian artery based on a case report and a review of the literature. J Thorac Cardiovasc Surg 1988;95: 888-891.

14. Gross C, Pressl F, Brücke P. Thoracic aneurysm in association with an aberrant right subclavian artery. Eur J Cardiothorac Surg 1990;4:105-106. 